\title{
Long-Term Oncologic Impact of Esophageal Anastomotic Leak After Esophagectomy
}

\section{Editorial on "Anastomotic Leak Does Not Impact on Long-Term Outcomes in Esophageal Cancer Patients” by Kamarajah SK, et al. (ASO-2019-09-1967.R1)}

\author{
Kimberly M. Linden, $\mathrm{MD}^{1}$, and David D. Shersher, $\mathrm{MD}^{2}$ \\ ${ }^{1}$ Department of Surgery, Cooper University Hospital, Camden, NJ; ${ }^{2}$ Department of Surgery, Division of Thoracic Surgery, \\ Cooper MD Anderson Cancer Center, Cooper University Hospital, Camden, NJ
}

Esophagectomies are associated with significant morbidity, involving both early and late postoperative complications. Anastomotic and chyle leaks, respiratory complications, and recurrent laryngeal nerve injury are considered early complications. Late complications include impaired conduit-emptying, reflux, dumping syndrome, and anastomotic stricture. ${ }^{1}$

Anastomotic leak rates vary from center to center, but in experienced high-quality programs, they should range between 5 and $20 \%$, with higher rates related to cervical anastomosis. $^{2}$ For intrathoracic anastomosis performed at high-quality centers, expected rates of leak should not exceed $10 \%$.

When leaks occur, they have a significant patient impact, realized by longer hospital and ICU stays as well as an increase in overall in-hospital mortality. However, a similar effect on the 1- or 2-year mortality rate is not nearly as demonstrable. ${ }^{3}$

Not all leaks are equal. Extrapolation of the impact that leak has on patient outcome first required development of a uniform nomenclature by the Esophagectomy Complications Consensus Group.

Anastomotic leak, defined as a full-thickness gastrointestinal defect involving the esophagus, anastomosis, staple line, or conduit irrespective of presentation or method of

(C) Society of Surgical Oncology 2020

First Received: 16 December 2019;

Published Online: 12 February 2020

D. D. Shersher, MD

e-mail: Shersher-david@cooperhealth.edu identification, is classified into three types as follows: type 1 (a local defect requiring no change in therapy or one treated medically), type 2 (a localized defect requiring interventional but not surgical therapy), and type 3 (a localized defect requiring surgical therapy). ${ }^{4}$ The clinical impact of leaks is classified using the Clavien-Dindo grading system and ranges from non-severe leaks (NSL) associated with grade 1 or 2 complications to several esophageal anastomotic leaks (SEALs) associated with grade 3 or 4 complications.

Clinical outcome differences with higher gradations of leak have been well described. Markar et al. ${ }^{5}$ reported a $28 \%$ greater overall likelihood of death for patients with SEAL from a study of 2944 patients in a French multiinstitution university database. For patients with NSL, however, no differences were appreciated. Consistent with other studies, it is clear that significant leaks are associated with major long-term risk.

Oncology-specific correlation of long-term risk after esophageal leak has been more difficult to describe due to multiple confounding variables in a complex disease process. Andreou et al. ${ }^{6}$ evaluated long-term survival after gastric and esophageal resection with anastomotic leak and concluded that patients with leak statistically had decreased overall survival, with a 5-year survival rate of $39 \%$ compared with $61 \%$ for patients without leak, and this was independent of tumor stage or biology.

Markar et al. $^{5}$ also associated SEAL with overall, locoregional, and mixed cancer recurrence, but interestingly, not with distant recurrence. Mirnezami et al. ${ }^{7}$ 
reported similar findings in colorectal patients who had anastomotic leaks, with higher overall and cancer-specific morality as well as higher local recurrence rates.

Could anastomotic leak represent a conduit of egress for cancer cells to spread to the mediastinum and potentiate locoregional recurrence? This, together with a parallel hypothesis of interleukin and cytokine proliferation, may be contributory to recurrence, but these theories are far from validated in actual practice. ${ }^{5-8}$ Especially surprising in many of these multicenter studies is the lack of significant difference in metastatic recurrence, which still is the most common cause of death from recalcitrant esophageal cancer.

An alternate hypothesis to locoregional recurrence after esophageal leak is inadequate negative oncologic margin, submucosal satellite lesions outside the field of radiation and surgery, or incomplete radical two- or three-field lymphadenectomy. Innumerable studies show that higherquality oncologic esophageal resection with better outcomes is performed at higher-volume, higher-quality, or more-experienced centers, and it may be the inclusion of lower-volume, less-experienced, or lower-quality programs in the aforementioned studies that confounds and obscures reported theories of recurrence.

We applaud Kamarajah et al. ${ }^{9}$ for changing the focus from multicenter study with all tiers of experience included in esophageal surgery to a single high-volume study focused on oncologic outcome after anastomotic leaks. These authors retrospectively identified 1063 patients during 20 years who underwent a two-stage, two-field transthoracic esophagectomy and evaluated clinicopathologic characteristics as well as oncologic and postoperative outcomes for the patients. Whereas 53\% of the patients received some form of neoadjuvant treatment, only $19 \%$ had squamous cell carcinoma histology. Cases were stratified into no-anastomotic leak (NSL) or SEAL. Lymphadenectomy quantity (23 to 29 nodes resected), morbidity (67\%), anastomotic leaks (8\%), and 30-day mortality (3\%) reflect quality data points comparable with those of other high-quality centers that specialize in esophageal surgery. 9,10

Of the 87 patients (8\%) who experienced anastomotic leaks, $45 \%$ experienced a SEAL, with only two deaths reported, both occurring within 30 days and consisting of $5 \%$ of all patients who died during this period. A total of 38 patients died in the hospital postoperatively, and 28 of these patient deaths occurred within 30 days after index surgery. The majority of the patients who died either in the hospital or within 30 days after surgery had no reported anastomotic leak. Additional shorter-term (60- and 90-day) leak-specific morbidity and mortality data were not further sub-characterized and were reported in the long-term survival and recurrence results presented by the authors. Not surprisingly, severe esophageal anastomotic leaks were associated with a significantly longer critical care and overall hospital stays than non-severe leaks $(p<0.001)$ or no leaks $(p=0.028)$.

In contrast to the aforementioned studies, Kamarajah et al. ${ }^{9}$ demonstrated no statistical difference in overall survival or recurrence-free survival between the cohort with leak and the cohort without leak. This finding also was upheld in the subgroup analysis of patients who received neoadjuvant therapy followed by surgery, a group similar to the patients treated currently with trimodality therapy.

The concept of a high-quality center for esophageal cancer surgery is the critical differentiator in this report. Whereas single-center studies often are overshadowed by multicenter collaborations, Kamarajah et al. ${ }^{9}$ purposefully used their high-volume and high-quality experience to compose an excellent single-institution study. Significant anastomotic leaks were few, and R1 resection was uncommon. Lymphadenectomy was complete, and 30-day mortality was lower than $4 \%$. As with other reports from high-quality centers, theirs is associated with good outcomes in both the short- and long terms, with the latter irrespective of anastomotic leak. Perhaps Kamarajah et al. ${ }^{9}$ indirectly exposed the age-old concept that complex, standardized procedures with multiple complications are best managed in centers with experience and expertise, and that correlating these complications with survival end points can certainly be done well in a single-institution study.

DISCLOSURE There are no conflicts of interest.

\section{REFERENCES}

1. Bolliger M, Kroehnert JA, Molineus F, Kandioler D, Schindl M, Riss P. Experiences with the standardized classification of surgical complications (Clavien-Dindo) in general surgery patients. Eur Surg. 2018;50:256-61.

2. Chin CS, Linden PA, Al-Dameh A, Swanson SJ. Esophageal resection and replacement. In: Pedro del Nido, Sellke FW, Swanson SJ, editors. Sabiston \& spencer surgery of the chest, 9th ed. Philadelphia: Elsevier; 2016, pp. 657-87.

3. Junemann-Ramirez M, Awan MY, Khan ZM, Rahamim JS. Anastomotic leakage post-esophagogastrectomy for esophageal carcinoma: retrospective analysis of predictive factors, management, and influence on long-term survival in a high-volume centre. Eur J Cardiothorac Surg. 2005;27:3-7.

4. Low DE, Alderson D, Cecconello I, Chang AC, Darling GE, DJourno $\mathrm{XB}$, et al. International consensus on standardization of data collection for complications associated with esophagectomy: Esophagectomy Complications Consensus Group (ECCG). Ann Surg. 2015;262:286-94.

5. Markar S, Gronnier C, Duhamel A, Mabrut JY, Bail JP, Carrere $\mathrm{N}$, et al. The impact of severe anastomotic leak on long-term survival and cancer recurrence after surgical resection for esophageal malignancy. Ann Surg. 2015;262:972-80. 
6. Andreou A, Biebl M, Dadras M, Struecker B, Sauer IM, ThussPatience PC, et al. Anastomotic leak predicts diminished longterm survival after resection for gastric and esophageal cancer. Surgery. 2016;160:191-203.

7. Mirnezami A, Mirnezami R, Chandrakumaran K, Sasapu K, Sagar P, Finan P. Increased local recurrence and reduced survival from colorectal cancer following anastomotic leak: systemic review and meta-analysis. Ann Surg. 2011;253:890-9.

8. Eberhardt JM, Kiran RP, Lavery IC. The impact of anastomotic leak and intraabdominal abscess on cancer-related outcomes after resection for colorectal cancer: a case-control study. Dis Colon Rectum. 2009;52:380-6.

9. Kamarajah SK, Navidi M, Wahed S, Immanuel A, Hayes N, Griffin SM, et al. Anastomotic leak does not impact on long-term outcomes in esophageal cancer patients. Ann Surg Oncol. 2020. https://doi.org/10.1245/s10434-020-08199-x.

10. Aurello P, Berardi G, Moschetta G, Cinquepalmi M, Antolino L, Nigri G, et al. Recurrence following anastomotic leakage after surgery for carcinoma of the distal esophagus and gastroesophageal junction: a systematic review. Anticancer Res. 2019;39:1651-60.

Publisher's Note Springer Nature remains neutral with regard to jurisdictional claims in published maps and institutional affiliations. 